\title{
Correction to: Association of Antihypertensive Agents with the Risk of In-Hospital Death in Patients with Covid-19
}

\author{
Laurent Chouchana ${ }^{1} \cdot$ Nathanaël Beeker ${ }^{2}$ - Nicolas Garcelon ${ }^{3,4}$. Bastien Rance ${ }^{3,5}$ - Nicolas Paris ${ }^{6}$. Elisa Salamanca ${ }^{6}$. \\ Elisabeth Polard ${ }^{7}$. Anita Burgun ${ }^{3,5,8}$. Jean-Marc Treluyer ${ }^{1,2}$. Antoine Neuraz ${ }^{3,8}$ - on behalf of AP-HP/Universities/ \\ Inserm COVID-19 research collaboration, AP-HP Covid CDR Initiative, and "Entrepôt de Données de Santé" AP-HP \\ Consortium
}

Published online: 4 March 2021

(C) Springer Science+Business Media, LLC, part of Springer Nature 2021

\section{Correction to: Cardiovascular Drugs and Therapy https://doi.org/10.1007/s10557-021-07155-5}

The original article has been corrected. The author group was incorrectly presented. Thus, the sentence fragments "all the persons below" and "are NOT authors" should be removed.

Publisher's Note Springer Nature remains neutral with regard to jurisdictional claims in published maps and institutional affiliations.

The online version of the original article can be found at https://doi.org/ $10.1007 / \mathrm{s} 10557-021-07155-5$

Laurent Chouchana

laurent.chouchana@aphp.fr

1 Centre Régional de Pharmacovigilance, Département de, Pharmacologie, Hôpital Cochin, AP-HP.Centre - Université de Paris, 27 rue du Faubourg Saint-Jacques, 75014 Paris, France

2 Unité de Recherche clinique, Hôpital Cochin, AP-HP.Centre Université de Paris, Paris, France

3 Centre de Recherche des Cordeliers, INSERM UMRS 1138 Team, 22, Université de Paris, Paris, France
4 Institut Imagine, Université de Paris, Paris, France

5 Département d'informatique médicale, Hôpital Européen Georges Pompidou, AP-HP.Centre - Université de Paris, Paris, France

6 Département Web Innovation Données (WIND), Direction des systèmes d'information, AP-HP, Paris, France

7 Centre Régional de Pharmacovigilance, pharmacoépidémiologie et information sur le médicament, CHU Rennes, Rennes, France

8 Département d'informatique médicale, Hôpital Necker-Enfants Malades, AP-HP.Centre - Université de Paris, Paris, France 